\title{
The Topical Tranexamic Acid Have Potential Hazard of Promoting Biofilm Formation of Staphylococcus aureus in Microenvironment of the Prosthetic Joint
}

\author{
Feiyang Zhang $\mathbb{D},{ }^{1}$ Wenjun Dong $\mathbb{D},{ }^{1}$ Fengyan Wang $\mathbb{D},{ }^{1}$ Jinlong $Y u\left(\mathbb{D},,^{1}\right.$ Feng Jiang $\mathbb{D}{ }^{1}$ \\ Jin Tang $\left(\mathbb{1},{ }^{2}\right.$ Yan Qian $\left(\mathbb{0},{ }^{1}\right.$ and Hao Shen ${ }^{1}{ }^{1}$ \\ ${ }^{1}$ Department of Orthopaedics, Shanghai Jiao Tong University Affiliated Sixth People's Hospital, \#600, Yishan Rd, Shanghai, China \\ ${ }^{2}$ Department of Clinical Laboratory, Shanghai Jiao Tong University Affiliated Sixth People's Hospital, \#600, Yishan Rd, \\ Shanghai, China
}

Correspondence should be addressed to Jin Tang; tangjin6ph@163.com, Yan Qian; yanzilaoma@126.com, and Hao Shen; shenhao7212@sina.com

Received 11 August 2020; Revised 1 February 2021; Accepted 4 March 2021; Published 12 March 2021

Academic Editor: Yu Sheng Li

Copyright ( 2021 Feiyang Zhang et al. This is an open access article distributed under the Creative Commons Attribution License, which permits unrestricted use, distribution, and reproduction in any medium, provided the original work is properly cited.

Background. Perioperative topical tranexamic acid as antifibrinolytic agent is often used for total joint replacement to reduce bleeding currently. Staphylococcus aureus was the most common isolates from perioperative infection of prosthetic joint. The influence of topical application with tranexamic acid on the incidence of acute prosthetic joint infection of Staphylococcus aureus has not been clarified. Methods. Mouse model of Staphylococcus aureus knee prosthesis infection was constructed. Tranexamic acid was intra-articular injected during the perioperative period. CFU counting from tissue and implant sample was evaluated 3 days and 7 days after inoculating of Staphylococcus aureus. Bacterial growth curve, biofilm formation, aggregation, and plasmin inhibition of Staphylococcus aureus were tested with tranexamic acid added to the synovial culture medium. Results. There were no significant differences of CFU counting from tissue and implant samples in knee prosthesis infection after a single local injection of tranexamic acid at the postoperative 3 or 7 days. The amount of bacterial colonization on the surface of implant increased after 3 days' continuous local injection of tranexamic acid. Tranexamic acid has no effect on bacterial growth at the concentration $(10 \mathrm{mg} / \mathrm{ml})$ of clinical application, but it can inhibit bacterial aggregation and mildly inhibit biofilm formation. Plasmin can significantly inhibit biofilm formation which can be revised by adding tranexamic acid. Conclusion. Although continuous local injection of tranexamic acid can promote the biofilm formation of Staphylococcus aureus on the surface of articular implant, it has clinical safety for using one single local injection of tranexamic acid during the perioperative period.

\section{Introduction}

Tranexamic acid can competitively inhibit the activation of plasminogen and the binding of plasmin to fibrin as synthetic analogues of the amino acid lysine, thus inhibiting fibrin degradation $[1,2]$. A large number of studies have confirmed that tranexamic acid can significantly reduce the amount of blood loss during surgery without increasing the risk of thromboembolism [3-6]. Intravenous administration and local injection of tranexamic acid are usually used in the perioperative period of total joint replacement [7]. A large num- ber of clinical studies showed that tranexamic acid can significantly reduce the incidence of postoperative adverse events $[4,8]$.

Prosthetic joint infection is considered to be the most severe complication related to total joint replacement $[9$, 10]. Staphylococcus aureus was predominant pathogen in postoperative and acute prosthetic joint infection [10-12]. Because of the numerous virulence factors and the ability to form biofilm on the surfaces of implant, Staphylococcus aureus leads to more severe clinical presentation and worse prognosis [12]. 
Some studies has found that application of tranexamic acid can exacerbate infections of Staphylococcus aureus in mice model $[13,14]$.

But in clinical practice, the topical application of tranexamic acid has been used more and more widely and has not shown increasing of infection rate [15-19]. The influence of topical tranexamic acid on Staphylococcus aureus infection is still controversial.

Therefore, it needs to be clarified whether local injection of tranexamic acid promotes the formation of biofilm and leads to the increased risk of Staphylococcus aureus infection.

\section{Materials and Methods}

2.1. Bacterial Strains, Reagents, and Ethics Statement. The strains used in this study were methicillin-susceptible Staphylococcus aureus (MSSA) ST1792 isolated from PJI prosthesis. The fluorescently label Staphylococcus aureus ST1792sfGFP which was preserved in our laboratory were used for confocal microscopic assay. The tranexamic acid (Aladdin, Shanghai, China) was used in in vivo and in vitro study of bacterial growth, aggregation, and biofilm formation. The plasmin (PLM, Sigma Aldrich, St Louis, MO, USA) was used in in vitro study of biofilm formation was purchased from and used at final concentrations of $100 \mu \mathrm{g} / \mathrm{ml}$. Synovial fluid (SF) was added to the medium and used in in vitro experiment. Collection of human synovial fluid from patients with osteoarthritis was approved by the Institutional Review Board of Shanghai Jiao Tong University Affiliated Sixth People's Hospital. Because synovial fluid was aspirated as part of the routine procedure before injection of hyaluronic acid and would have been discarded otherwise, and patient information not collected, a waiver for informed written consent was granted by the IRB.

2.2. Staphylococcus aureus Prosthetic Joint Infection Mouse Model. All procedures of animal experiment had been reviewed and approved by the Animal Care and Experiment Committee of Shanghai Jiao Tong University affiliated Sixth People's Hospital.

Six-week-old and weighing $(20 \pm 5) \mathrm{g}$ male C57BL/6 mice were used to construct Staphylococcus aureus prosthetic joint infection model. The mice were anesthetized intraperitoneally with $3 \%$ pentobarbital sodium $(0.1 \mathrm{ml}$ per $10 \mathrm{~g}$ body weight) and then shaving the both knees. A medial parapatellar incision was made at the knee joint of mice after sterilized with 75\% alcohol. Proximal tibia was exposed, and the medullary cavity was inserted with sterile stainless steel implant rods. After closing the wounds by sutures, tranexamic acid was injected into the joint cavity of one side at a dose of $10 \mathrm{mg} / \mathrm{kg}$; the other side was injected with an equal volume of saline as control. Two hours later, both knee joints were inoculated with ST1792 solution of $\sim 5 * 10^{6}$. And then, the mice were randomly divided into three groups (Table 1). The first group was housed for 3 days, and the second group was housed for 7 days. The third group was continuous local injected with tranexamic acid at a dose of $10 \mathrm{mg} / \mathrm{kg}$ every 24 hours and housed for 3 days. Three days and 7 days after surgery, the mice with a single local injection of tranexamic acid (i.e., first and second groups) were euthanized, and the mice with continuous local injection of tranexamic acid were euthanized in 3 days after surgery. Peri-implant tissues and implant in tibia were harvested for CFU counting.

2.3. Bacteria CFU Counting. The peri-implant tissues were weighed and homogenized in tubes with $1 \mathrm{ml}$ normal of saline (NS) by high-speed homogenizer (Jingxin Industrial Limited Company, Shanghai, China). The implant were washed gently 3 times with NS to remove the planktonic bacteria and placed in tube containing $1 \mathrm{ml}$ of NS, sonicated, and homogenized to detach the adherent bacteria. The homogenates and suspensions were serially diluted in NS and spread on sheep blood agar (SBA). The plates were cultured overnight at $37^{\circ} \mathrm{C}$. The colonies were counted and expressed by $\log _{10} \mathrm{CFU} / \mathrm{g}$ of peri-implant tissues and $\log _{10} \mathrm{CFU} /$ per implanted rods.

2.4. The Effect Test of TXA In Vitro. The ST1792 were cultured overnight at $37^{\circ} \mathrm{C}$ on SBA plates before each experiment. Single colony of each strain was collected and cultured overnight in TSB at $37^{\circ} \mathrm{C}$. The ST1792-sfGFP were cultured on Tryptic Soy Agar (TSA) plates with $10 \mu \mathrm{g} / \mathrm{ml}$ chloramphenicol and cultured overnight in TSB containing $10 \mu \mathrm{g} / \mathrm{ml}$ chloramphenicol at $37^{\circ} \mathrm{C}$.

The preconditioning method of medium was divided into six groups: the first group containing $10 \% \mathrm{SF}$, the second group containing $10 \% \mathrm{SF}$ and $10 \mathrm{mg} / \mathrm{ml} \mathrm{TXA}$, the third group containing $10 \% \mathrm{SF}$ and $50 \mathrm{mg} / \mathrm{ml} \mathrm{TXA}$, the fourth group containing $10 \% \mathrm{SF}$ and $100 \mu \mathrm{g} / \mathrm{ml}$ PLM, the fifth group containing $10 \% \mathrm{SF}$ and $100 \mu \mathrm{g} / \mathrm{ml}$ PLM and $10 \mathrm{mg} / \mathrm{ml}$ TXA, and the sixth group containing $10 \% \mathrm{SF}$ and $100 \mu \mathrm{g} / \mathrm{ml}$ PLM and $50 \mathrm{mg} / \mathrm{ml}$ TXA.

2.4.1. Staphylococcus aureus Growth Curve Assay. Overnight culture of ST1792 was serially diluted $1: 1000$ in groups 1, 2, and 3 with TSB and cultured at $37^{\circ} \mathrm{C} .100 \mu \mathrm{l}$ of solution were aspirated at 0-2-4-6-8-12 hours and transferred to the wells of a 96-well tissue culture plate (Corning Co., NY, USA). The absorbance was measured by a microplate reader (BIO-TEK, ELX 800) at a wavelength of $600 \mathrm{~nm}$.

2.4.2. Staphylococcus aureus Aggregation Test. Overnight culture of ST1792 was serially diluted to $1: 1000$ in groups 1, 2, and 3 with TSB and three additional control groups without $10 \% \mathrm{SF}$ and cultured at $37^{\circ} \mathrm{C}$. Strain clumped together and sank to the bottom of the tube after $8 \mathrm{~h}$; the turbidity of medium supernatant was significantly decreased. $100 \mu \mathrm{l}$ of culture medium supernatant were aspirated and transferred to the wells of a 96-well tissue culture plate, measuring the absorbance at a wavelength of $490 \mathrm{~nm}$ by a microplate reader.

2.4.3. Biofilm Formation and Biomass. Overnight culture of ST1792 was serially diluted to $1: 1000$ in TSB supplemented with $0.5 \%$ glucose (TSBG). Diluted bacteria, with supplementation from group 1 to group 6, were used for assessment of biofilm formation on (1) the bottom of 96-well polystyrene microtiter plate (Corning Co., NY, USA) or (2) Ultra High Molecular Weight Polyethylene (UHMWPE) washers that 
TABLE 1: The grouping of mouse.

\begin{tabular}{lccc}
\hline Group & Left knee & Treatment & Right knee \\
\hline 1 & A single local injection of TXA & A single local injection of NS & Time (day) \\
2 & A single local injection of TXA & A single local injection of NS & 3 \\
3 & Continuous local injection of TXA & Continuous local injection of NS & 7 \\
\hline
\end{tabular}

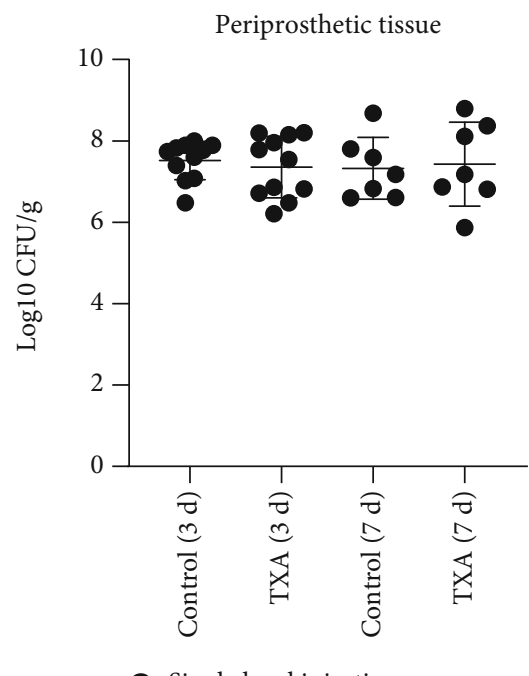

(a)

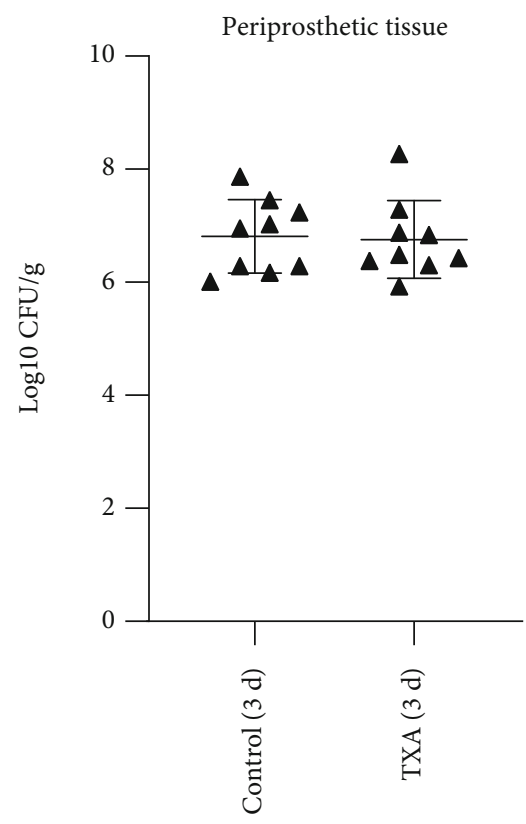

A Continuous local injection

(c)

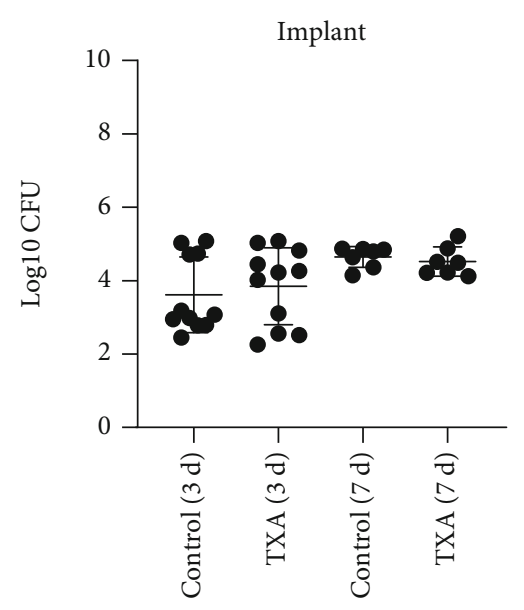

Single local injection

(b)

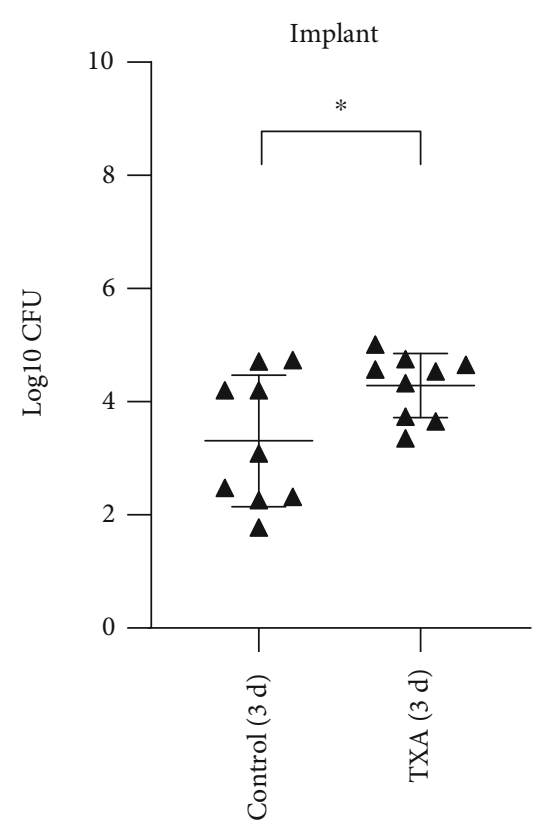

A Continuous local injection

(d)

FIgURE 1: CFU counting after local injection of tranexamic acid on Staphylococcus aureus PJI mouse model. (a, b) The bacterial CFU of PJI mice with a single local injection of tranexamic acid in periprosthetic tissue (Figure 2(a)) and implants (Figure 2(b)) after 3 and 7 days. (c) The bacterial CFU of periprosthetic tissue after continuous local injection of tranexamic acid for 3 days. (d) The bacterial CFU of implants after continuous local injection of tranexamic acid for 3 days. ${ }^{*} P<0.05$; error bars represent standard deviations. 
were affixed to bottom of 24-well polystyrene plates (Corning Co., NY, USA) with Lubriseal grease (Thomas Scientific) and sterilized by UV irradiation.

96-well plate were incubated overnight at $37^{\circ} \mathrm{C}$. Completely aspiring the supernatant from each well and washing gently 3 times with $200 \mu \mathrm{l}$ NS to remove the planktonic bacteria. Then, $100 \mu \mathrm{l}$ methanol was used to fix the biofilm for $30 \mathrm{~min}$ and then dried. After that, biofilm was stained with $100 \mu \mathrm{l}$ of $0.1 \%$ crystal violet for $15 \mathrm{~min}$. The biofilm biomass on the bottom of the well was dissolved in $200 \mu \mathrm{l} 33 \%$ acetic acid after the unbound crystal violet was rinsed by NS for 3 times. $100 \mu \mathrm{l}$ solution of each well were transferred into a new 96-well tissue culture plate. The absorbance was measured by a microplate reader at a wavelength of $590 \mathrm{~nm}$.

UHMWPE washer inoculated with ST1792 in groups 1, $2,3,4,5$, and 6 with TSBG was incubated overnight. The UHMWPE washers were washed 3 times with saline to remove nonadherent cells. The washed UHMWPE washers were transferred into a new 24-well polystyrene plate. Adherent biofilms were fixed with methanol and stained with crystal violet and washed 3 times with sterile water. Biomass on the surfaces of UHMWPE washers was determined by solubilizing crystal violet with $33 \%$ acetic acid as described above.

2.4.4. Confocal Laser Scanning Microscopy (CLSM). Overnight culture of ST1792-sfGFP was serially diluted $1: 1000$ in groups $1,2,3,4,5$, and 6 with TSBG. $500 \mu \mathrm{l}$ of bacteria suspension were incubated with cover glass overnight in 24-well tissue culture plate at $37^{\circ} \mathrm{C}$. The cover glasses were washed gently 3 times with NS and imaged by CLSM. The ST1792sfGFP showed green fluorescence.

\section{Results}

3.1. The Colonization on the Surface of Implant Were Increased by Continuous Use with TXA In Vivo. Mice with a single local injection of tranexamic acid during the perioperative period showed no significant difference in Staphylococcus aureus colonization of tissue and implant sample after 3 and 7 days (Figures 1(a) and 1(b)). Although mice with continuous local injection of tranexamic acid for 3 days showed no significant increase of bacterial colonization in tissue (Figure 1(c)), there was significant higher CFU counting from implant (Figure 1(d)). The experimental results suggested that the continuous local injection of tranexamic acid can promote biofilm formation.

\subsection{Influence of Tranexamic Acid on the Growth of} Staphylococcus aureus. For analysis of the influence of tranexamic acid on bacterial growth curve, tranexamic acid with clinically used concentration $(10 \mathrm{mg} / \mathrm{ml})$ and high concentration $(50 \mathrm{mg} / \mathrm{ml})$ was used. Compared to the medium without tranexamic acid, the medium containing tranexamic acid with clinically used concentration $(10 \mathrm{mg} / \mathrm{ml})$ has no influence on bacterial growth. Bacterial growth was inhibited by tranexamic acid with high concentration $(50 \mathrm{mg} / \mathrm{ml})$ (Figure 2). It showed that tranexamic acid has no antibacterial capacity in concentration of clinical use.

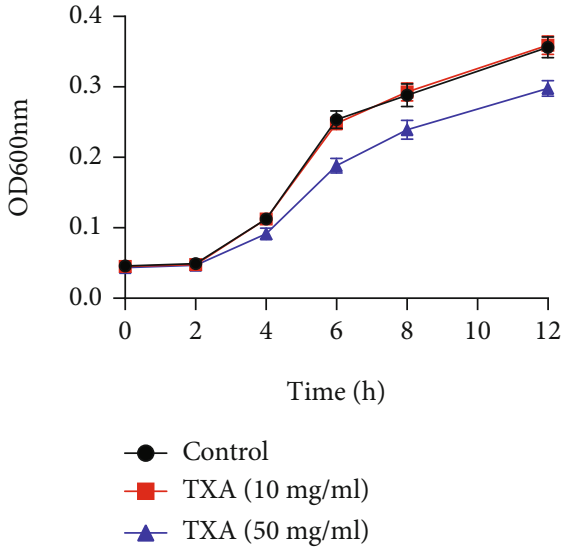

Figure 2: Bacterial growth curve of Staphylococcus aureus ST1792 cultured separately in TSB medium containing $10 \%$ SF (Blank,10 mg/ml TXA and $50 \mathrm{mg} / \mathrm{ml}$ TXA).

3.3. The Influence of Tranexamic Acid on the Bacterial Aggregation in Different Media. The aggregation of Staphylococcus aureus inhibited by tranexamic acid with clinically used concentration $(10 \mathrm{mg} / \mathrm{ml})$ in TSB medium. The inhibitory effect was attenuated in tranexamic acid with high concentration $(50 \mathrm{mg} / \mathrm{ml}$ ) (Figure 3(a)). However, the antiaggregation capacity of tranexamic acid disappears when cultured in TSB medium containing 10\% SF(Figure 3(b)). The results suggested that inhibitory effect of tranexamic acid on bacterial aggregation attenuated in the microenvironment of the prosthetic joint.

3.4. The Individual and Mutual Inhibition for Biofilm Formation of Staphylococcus aureus by Tranexamic Acid and Plasmin. Whether on 96-well plates or UHMWPE washer, the biofilm formation of Staphylococcus aureus was slight inhibited by tranexamic acid (Figures 4(a) and 4(b)). Biofilm formation were inhibited more significantly by tranexamic acid in high concentration $(50 \mathrm{mg} / \mathrm{ml})$ with the inhibitory ability of bacterial growth. When plasmin was added into the culture medium, the biofilm formation was significantly inhibited. However, the antibiofilm formation ability of plasmin inhibited by tranexamic acid; thus, the culture medium exhibited a weak antibiofilm formation effect.

3.5. Tranexamic Acid Offset the Inhibition of Biofilm Formation with Plasmin via CLSM. Biofilm formed obviously in TSBG with $10 \%$ SF by ST1792 and slight inhibited with TXA $(10 \mathrm{mg} / \mathrm{ml})$. The inhibitory effect of TXA at high concentration $(50 \mathrm{mg} / \mathrm{ml})$ was more obvious. However, the biofilm disappeared after PLM was added, and it can be found that antibiofilm formation function of plasmin much more strong than TXA in TSBG with 10\%SF (Figure 5). The weak antibiofilm formation function exhibited in medium when acting simultaneously with tranexamic acid and plasmin. The results of CLSM provide further confirmation that tranexamic acid offset the inhibition of biofilm formation with plasmin. 


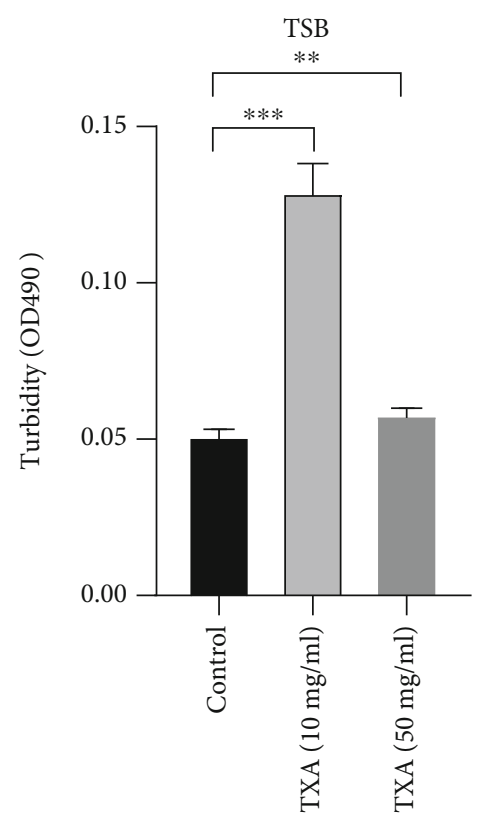

(a)

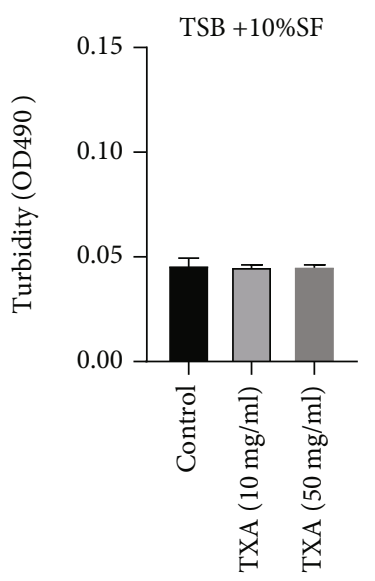

(b)

FIgURE 3: Tranexamic acid shows difference of inhibiting bacterial aggregation in TSB with or without SF. (a) The turbidity of medium supernatant increased in TSB medium containing TXA $(n=6)$. (b) No significant difference of the turbidity was observed in TSB medium containing SF and TXA. ${ }^{* *} P<0.01,{ }^{* * *} P<0.001$. Error bars represent standard deviations.

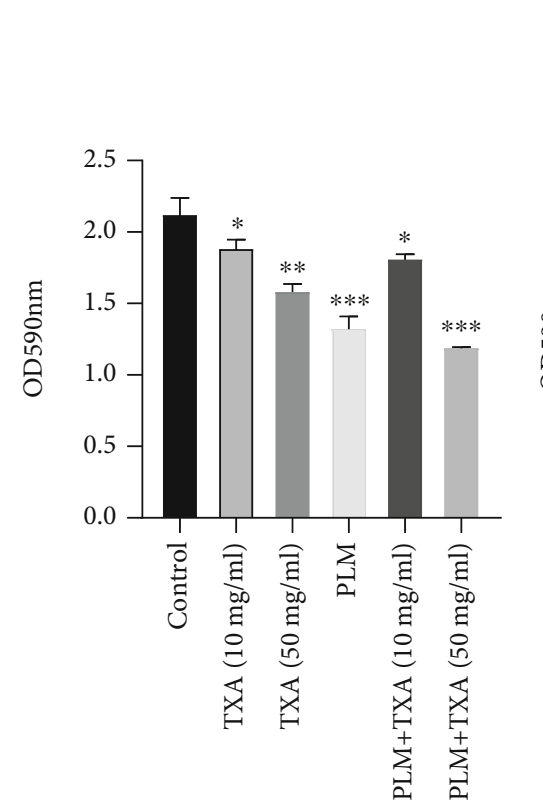

(a)
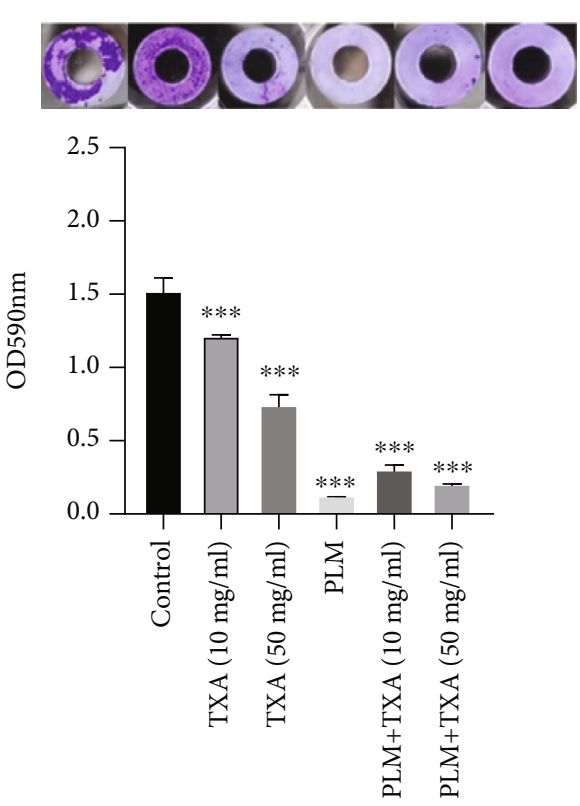

(b)

FIGURE 4: Inhibitory effect of biofilm formation with tranexamic acid were observed in 96-well tissue culture plate (Figure 4(a)) and on the surface of UHMWPE washer (Figure 5(b)). Biofilm formation inhibited more obviously by plasmin and the inhibition of plasmin were reversed by tranexamic acid. ${ }^{*} P<0.05,{ }^{* *} P<0.01,{ }^{* * *} P<0.001$. Error bars represent standard deviations.

\section{Discussion}

Mice studies with staphylococcal sepsis or septic arthritis showed increasing severity and mortality of infection by treatment with tranexamic acid; however, the mice were infused with tranexamic acid at a dose of $700-800 \mathrm{mg} / \mathrm{kg}$ every 8 hours [14] which was much higher dosage than that for clinical use [14], and previous study had proposed that high-dose tranexamic acid caused significant cytotoxicity [20]. In present study, we constructed Staphylococcus aureus 


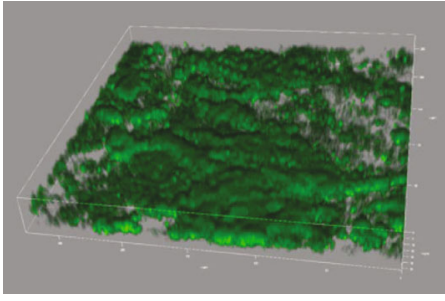

TSBG+10\%SF

(a)

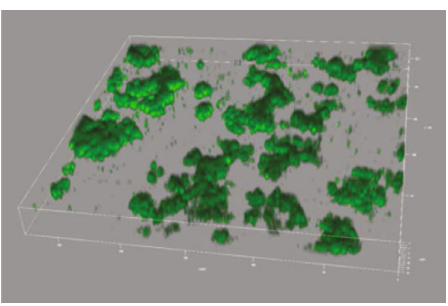

TSBG+10\%SF+TXA $(50 \mathrm{mg} / \mathrm{ml})$

(c)

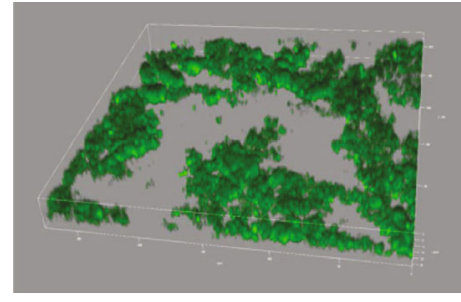

TSBG+10\%SF+TXA $(10 \mathrm{mg} / \mathrm{ml})+\mathrm{PLM}$

(e)

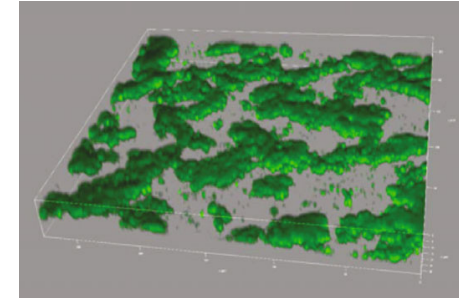

TSBG+10\%SF+TXA $(10 \mathrm{mg} / \mathrm{ml})$

(b)

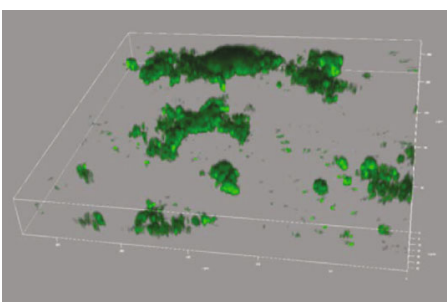

TSBG+10\%SF+PLM

(d)

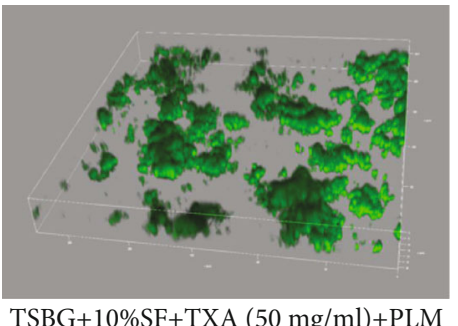

(f)

FIGURE 5: CLSM results of biofilm forming with ST1792-sfGFP. (a) Biofilm formation of Staphylococcus aureus in TSBG medium containing $10 \%$ SF. (b) Biofilm formation was inhibited by tranexamic acid $(10 \mathrm{mg} / \mathrm{ml})$. (c) Biofilm formation was significantly inhibited by tranexamic acid $(50 \mathrm{mg} / \mathrm{ml})$. (d) Biofilm formation was significantly inhibited by plasmin. (e, f) Tranexamic acid offset the inhibition of biofilm formation with plasmin.

prosthetic joint infection model with topical tranexamic acid at $10 \mathrm{mg} / \mathrm{kg}$ which dose was similar with that using clinically and found that continuous local injection of tranexamic acid promoted biofilm formation in vivo.

Clinically, a single local injection of tranexamic acid is commonly used in joint replacement surgery, and the safety of this method is well documented $[15,16,18]$. In our in vivo experiments, it was observed that a single local injection of tranexamic acid did not aggravate Staphylococcus aureus infection. However, for patients bleeding after joint implanted surgery, we hope to explore the feasibility of continuous local injection of tranexamic acid. With the help of mice model, continuous local injection of tranexamic acid was observed to promote the formation of $S$. aureus biofilm, which is the potential risk of using tranexamic acid. Continuous local injection of tranexamic acid is not recommended for clinical treatment.

In order to clarify action of tranexamic acid against Staphylococcus aureus, bacterial growth, aggregation, and biofilm formation has been studied in vitro experiment. Results indicated no influence of tranexamic acid with clinical concentration $(10 \mathrm{mg} / \mathrm{ml})$ on Staphylococcus aureus growth. However, tranexamic acid showed inhibitory function on aggregation and biofilm formation of Staphylococcus aureus. The function of antibacterial aggregation disappeared in microenvironment containing synovial fluid. High concentration of TXA $(50 \mathrm{mg} / \mathrm{ml})$ showed inhibition of bacterial growth and relatively stronger function of antibiofilm formation, but the significant cytotoxicity exhibited in the high concentration of TXA which was not used in clinically cases [20].

We can observe some special phenomenon in bacteria aggregation experiments. In the infected setting, polysaccharide-based aggregates and biofilms can be regarded as different phases of the same process. Aggregate seeding biofilms, while biofilms dispersing into free floating aggregates [21]. The aggregates can enhance the protection from phagocytosis and be more tolerant to antibiotic treatment [22]. Therefore, the supernatant of bacteria in TSB with TXA had higher turbidity, which proves that the clinical concentration of TXA can inhibit bacteria aggregation, and the high concentration of TXA can inhibit the growth of Staphylococcus aureus, resulting in lower turbidity. However, tranexamic acid's inhibitory ability on bacterial aggregation 
disappeared in synovial fluid environment, which may be related to the complex joint microenvironment, and further research is needed.

The primary target of action for tranexamic acid was plasmin [2]. The double-sided effect of plasmin in the process of Staphylococcus aureus infection has been shown in previous studies. On one hand, plasmin can act in a proinflammatory manner via triggering chemotaxis and cytokine release [23], and the histones in neutrophil extracellular traps (NETs) cleaved by plasmin, which lead to protection of Staphylococcus aureus in vivo [24]. Therefore, plasmin showed the function of promoting infection. On the other hand, fibrin-containing bacterial biofilms were decomposed by the specifically targeting fibrin function of plasmin, and the antibiotic efficiency were greatly improved [22, 25]. Thus, plasmin showed the function of inhibiting infection.

In this study, the significant antibiofilm function of plasmin was found in experiments compared with tranexamic acid. The obvious inhibitory effect of plasmin on Staphylococcus aureus biofilm formation was mentioned in previous studies [22], but this significant antibiofilm function of plasmin inhibited by tranexamic acid. Therefore, the increased colonization of Staphylococcus aureus made it easier to form biofilms by tranexamic acid on the surface of implant. While tranexamic acid promoted biofilm by inhibiting plasmin, it also inhibited the degradation of histones in NETs. Therefore, one single local injection has not shown to promote infection in vivo, but continuous use of tranexamic acid in vivo has shown increased Staphylococcus aureus colonization, which suggested the potential risk of topical tranexamic acid.

There are still some limitations in this study. Although the clinical used concentration of tranexamic acid has been applied to avoid the cytotoxicity caused by high concentration, the viewing time of mouse model was relatively short and the effect of continuous topical tranexamic acid for a longer time has not been tested. The influence of tranexamic acid on antibiotic sensitivity of Staphylococcus aureus has not been explored in the present study. Although Staphylococcus aureus ST1792 was used in terms of strains, the response of various Staphylococcus aureus subtypes to tranexamic acid has not been verified.

\section{Conclusions}

In summary, here, we clarified the function of topical tranexamic acid use with Staphylococcus aureus. Continuous topical injection of tranexamic acid promoted biofilm formation of Staphylococcus aureus which has potential risks to patients undergoing total joint replacement. Although it has clinical safety for using one single local injection of tranexamic acid, it is recommended to use antibiotics in combination with topical using of tranexamic acid in clinic to minimizing the rate of infection.

\section{Data Availability}

The data used to support the findings of this study are available from the corresponding author upon request.

\section{Conflicts of Interest}

The authors have no conflicts of interest.

\section{Authors' Contributions}

Zhang, Feiyang, Dong, Wenjun, and Wang, Fengyan contributed equally to this work.

\section{Acknowledgments}

This study was supported by National Natural Science Foundation of China (Grant no: 81772364) and Medical Guidance Scientific Research Support Project of Shanghai Science and Technology Commission (Grant no: 19411962600).

\section{References}

[1] C. Longstaff, "Studies on the mechanism of action of aprotinin and tranexamic acid as plasmin inhibitors and antifibrinolytic agents," Blood Coagulation \& Fibrinolysis: an International Journal in Haemostasis and Thrombosis, vol. 5, pp. 537-542, 1994.

[2] P. L. McCormack, "Tranexamic acid: a review of its use in the treatment of hyperfibrinolysis," Drugs, vol. 72, no. 5, pp. 585617, 2012.

[3] R. Gandhi, H. M. Evans, S. R. Mahomed, and N. N. Mahomed, "Tranexamic acid and the reduction of blood loss in total knee and hip arthroplasty: a meta-analysis," BMC Research Notes, vol. 6, no. 1, p. 184, 2013.

[4] J. Poeran, R. Rasul, S. Suzuki et al., "Tranexamic acid use and postoperative outcomes in patients undergoing total hip or knee arthroplasty in the United States: retrospective analysis of effectiveness and safety," BMJ, vol. 349, no. aug12 8, article g4829, 2014.

[5] J. T. Moskal and S. G. Capps, "Intra-articular tranexamic acid in primary total knee arthroplasty: meta-analysis," The Journal of Knee Surgery, vol. 31, no. 1, pp. 056-067, 2017.

[6] Z. Wang and X. Shen, "The efficacy of combined intraarticular and intravenous tranexamic acid for blood loss in primary total knee arthroplasty: a meta-analysis," Medicine, vol. 96, no. 42, article e8123, 2017.

[7] D. Gulabi, Y. Yuce, K. H. Erkal, N. Saglam, and S. Camur, "The combined administration of systemic and topical tranexamic acid for total hip arthroplasty: is it better than systemic?," Acta Orthopaedica et Traumatologica Turcica, vol. 53, no. 4, pp. 297-300, 2019.

[8] H. Yazdi, M. R. Klement, M. Hammad et al., "Tranexamic acid is associated with reduced periprosthetic joint infection after primary total joint arthroplasty," The Journal of Arthroplasty, vol. 35, no. 3, pp. 840-844, 2020.

[9] S. M. Kurtz, E. Lau, H. Watson, J. K. Schmier, and J. Parvizi, "Economic burden of periprosthetic joint infection in the United States," The Journal of Arthroplasty, vol. 27, no. 8, pp. 61-65.e1, 2012.

[10] J. L. Del Pozo and R. Patel, "Clinical practice. Infection associated with prosthetic joints," The New England Journal of Medicine, vol. 361, no. 8, pp. 787-794, 2009.

[11] V. K. Aggarwal, H. Bakhshi, N. U. Ecker, J. Parvizi, T. Gehrke, and D. Kendoff, "Organism profile in periprosthetic joint infection: pathogens differ at two arthroplasty infection 
referral centers in Europe and in the United States," The Journal of Knee Surgery, vol. 27, no. 5, pp. 399-406, 2014.

[12] M. Titécat, E. Senneville, F. Wallet et al., "Bacterial epidemiology of osteoarticular infections in a referent center: 10-year study," Orthopaedics \& Traumatology, Surgery \& Research, vol. 99, no. 6, pp. 653-658, 2013.

[13] D. F. Draxler, M. M. Awad, G. Hanafi et al., "Tranexamic acid influences the immune response, but not bacterial clearance in a model of post-traumatic brain injury pneumonia," Journal of Neurotrauma, vol. 36, no. 23, pp. 3297-3308, 2019.

[14] M. Kłak, N. Anäkkälä, W. Wang et al., “Tranexamic acid, an inhibitor of plasminogen activation, aggravates staphylococcal septic arthritis and sepsis," Scandinavian Journal of Infectious Diseases, vol. 42, no. 5, pp. 351-358, 2010.

[15] J. Y. Chen, N. N. Lo, D. K. Tay, P. L. Chin, S. L. Chia, and S. J. Yeo, "Intra-articular administration of tranexamic acid in total hip arthroplasty," Journal of Orthopaedic Surgery (Hong Kong), vol. 23, no. 2, pp. 213-217, 2015.

[16] J. S. Kang, K. H. Moon, B. S. Kim, and S. J. Yang, "Topical administration of tranexamic acid in hip arthroplasty," International Orthopaedics, vol. 41, no. 2, pp. 259-263, 2017.

[17] C. Wang, G. J. Xu, Z. Han et al., "Topical application of tranexamic acid in primary total hip arthroplasty: a systemic review and meta-analysis," International Journal of Surgery, vol. 15, pp. 134-139, 2015.

[18] B. S. Waddell, T. Zahoor, M. Meyer, L. Ochsner, and G. Chimento, "Topical tranexamic acid use in knee periprosthetic joint infection is safe and effective," The Journal of Knee Surgery, vol. 29, no. 5, pp. 423-429, 2016.

[19] F. Reichel, C. Peter, V. Ewerbeck, and M. Egermann, "Reducing blood loss in revision total hip and knee arthroplasty: tranexamic acid is effective in aseptic revisions and in second-stage reimplantations for periprosthetic infection," BioMed Research International, vol. 2018, Article ID 3891870, 9 pages, 2018.

[20] M. McLean, K. McCall, I. D. M. Smith et al., "Tranexamic acid toxicity in human periarticular tissues," Bone \& Joint Research, vol. 8, no. 1, pp. 11-18, 2019.

[21] H. A. Crosby, J. Kwiecinski, and A. R. Horswill, “_Staphylococcus aureus_aggregation and coagulation mechanisms, and their function in host-pathogen interactions," Advances in Applied Microbiology, vol. 96, pp. 1-41, 2016.

[22] S. Dastgheyb, J. Parvizi, I. M. Shapiro, N. J. Hickok, and M. Otto, "Effect of biofilms on recalcitrance of staphylococcal joint infection to antibiotic treatment," The Journal of Infectious Diseases, vol. 211, no. 4, pp. 641-650, 2015.

[23] D. F. Draxler and R. L. Medcalf, "The fibrinolytic system-more than fibrinolysis?," Transfusion Medicine Reviews, vol. 29, no. 2, pp. 102-109, 2015.

[24] G. Pietrocola, G. Nobile, M. J. Alfeo et al., "Fibronectin-binding protein $\mathrm{B}$ (FnBPB) from_Staphylococcus aureus_protects against the antimicrobial activity of histones," The Journal of Biological Chemistry, vol. 294, no. 10, pp. 3588-3602, 2019.

[25] Y. Guo, J. Li, E. Hagström, and T. Ny, "Protective effects of plasmin (ogen) in a mouse model of Staphylococcus aureusinduced arthritis," Arthritis and Rheumatism, vol. 58, no. 3, pp. 764-772, 2008. 